\title{
A Fusion Protein Consisting of the Vaccine Adjuvant Monophosphoryl Lipid A and the Allergen Ovalbumin Boosts Allergen-Specific Th1, Th2, and Th17 Responses In Vitro
}

\author{
Stefan Schülke, ${ }^{1}$ Lothar Vogel, ${ }^{2}$ Ann-Christine Junker, ${ }^{1}$ Kay-Martin Hanschmann, ${ }^{3}$ \\ Adam Flaczyk, ${ }^{1}$ Stefan Vieths, ${ }^{1}$ and Stephan Scheurer ${ }^{1}$ \\ ${ }^{1}$ Vice President's Research Group 1: Molecular Allergology, Paul-Ehrlich-Institut, Langen, Hessen, Germany \\ ${ }^{2}$ Division of Allergology, Paul-Ehrlich-Institut, 63225 Langen, Germany \\ ${ }^{3}$ Division of Microbiology, Paul-Ehrlich-Institut, 63225 Langen, Germany \\ Correspondence should be addressed to Stefan Schülke; stefan.schuelke@pei.de
}

Received 7 March 2016; Revised 25 April 2016; Accepted 17 May 2016

Academic Editor: Leandro J. Carreño

Copyright (C) 2016 Stefan Schülke et al. This is an open access article distributed under the Creative Commons Attribution License, which permits unrestricted use, distribution, and reproduction in any medium, provided the original work is properly cited.

Background. The detoxified TLR4-ligand Monophosphoryl Lipid A (MPLA) is the first approved TLR-agonist used as adjuvant in licensed vaccines but has not yet been explored as part of conjugated vaccines. Objective. To investigate the immune-modulating properties of a fusion protein consisting of MPLA and Ovalbumin (MPLA : Ova). Results. MPLA and Ova were chemically coupled by stable carbamate linkage. MPLA : Ova was highly pure without detectable product-related impurities by either noncoupled MPLA or Ova. Light scattering analysis revealed MPLA : Ova to be aggregated. Stimulation of mDC and mDC: DO11.10 CD4 ${ }^{+}$ TC cocultures showed a stronger activation of both $\mathrm{mDC}$ and Ova-specific DO11.10 CD4 ${ }^{+}$TC by MPLA : Ova compared to the mixture of both components. MPLA : Ova induced both strong proinflammatory (IL- $1 \beta$, IL- 6 , and TNF- $\alpha$ ) and anti-inflammatory (IL-10) cytokine responses from mDCs while also boosting allergen-specific Th1, Th2, and Th17 cytokine secretion. Conclusion. Conjugation of MPLA and antigen enhanced the immune response compared to the mixture of both components. Due to the nonbiased boost of Ova-specific Th2 and Th17 responses while also inducing Th1 responses, this fusion protein may not be a suitable vaccine candidate for allergy treatment but may hold potential for the treatment of other diseases that require a strong stimulation of the host's immune system (e.g., cancer).

\section{Introduction}

Currently, conventional allergen immunotherapy (AIT) with allergen extracts is not convenient for patients due to a multiyear treatment regimen [1]. For some allergies, AIT is only partially efficacious and can be hampered by unwanted side effects. To improve AIT, novel vaccine candidates and accompanying adjuvants that increase efficacy while decreasing unwanted adverse-effects are needed [2].

In this context, the discovery of TLR-ligands with their intrinsic ability to induce robust innate immune responses was thought to hold great potential for the discovery and development of novel adjuvants. One of the bestcharacterized TLR-ligands is lipopolysaccharide (LPS), a cell wall component of Gram-negative bacteria that activates
TLR4. Despite its strong immune-stimulatory potential, its use as an adjuvant is strongly limited due to its inherent toxicity [3]. Accordingly, nucleic acid-based TLR-ligands, such as CpG (TLR9), R848 (TLR7/8), or Poly I:C (TLR3), are good immune activators but are hampered in their clinical efficacy due to problems with both toxicity and stability in vivo [3].

To be able to take advantage of the strong immuneactivating properties of TLR-ligands without the inherent toxicity, variants of TLR-ligands were generated by chemical modification which should retain most of their immunestimulating properties [4]. One such adjuvant is the TLR4ligand Monophosphoryl Lipid A (MPLA), a detoxified LPSderivative. MPLA was derived from the LPS of Salmonella minnesota R595 by a series of organic extractions followed by 
mild acid and alkaline treatments [4]. This resulted in three distinct modifications compared to the parent molecule: (1) the removal of the core polysaccharide containing the $\mathrm{O}$ antigen, (2) the removal of one phosphate group, and (3) one fatty acid chain [4].

Up to now, MPLA is the only TLR-ligand used as an adjuvant in licensed vaccines. Several vaccines including Fendrix $^{\circledR}$ (hepatitis B), Cervarix ${ }^{\circledR}$ (human papillomavirus16 and papillomavirus-18), RTS, ${ }^{\circledR}$ (malaria) [5-7], and the allergen product Pollinex ${ }^{\circledR}$ Quattro (pollen allergies) [8] which contain MPLA as one component of more complex adjuvant systems have been licensed or are undergoing phase III clinical trials. Immunologically, MPLA has been repeatedly shown to induce a predominantly Thl-biased immune response [9]. The application of allergen therapeutics containing MPLA leads to isotype switching from allergenspecific $\mathrm{IgE}$ antibodies towards $\mathrm{IgG}_{1}$ - and $\mathrm{IgG}_{4}$-dominated humoral immune response in humans [10].

While MPLA was reported to be less toxic and pyrogenic than LPS [11], the recent approval of several vaccine formulations adjuvanted with MPLA prompted us to initiate further detailed investigations of its adjuvant potential. In a previous study we showed that, in direct comparison to LPS, MPLA-stimulation induced similar but attenuated immune responses in several important immune cell types such as mouse epithelial cells, myeloid dendritic cells (mDCs), B and $\mathrm{T}$ cells, and human ex vivo isolated monocytes. Interestingly, MPLA was not able to activate either human or mouse mast cells [12].

After initially characterizing MPLA's immune-activating potential [12] we wanted to determine its potential as an integral part of an adjuvant:allergen fusion protein. In several experimental allergy models, such conjugates, for example, incorporating TLR5-, TLR7/8-, and TLR9-ligands, have been described to have beneficial immune-modulating properties by promoting Th1- and appropriate regulatory responses [13-16].

To this end, we chemically coupled MPLA to the model allergen Ovalbumin (Ova) and characterized the conjugate by SDS-PAGE and light scattering analysis. Subsequently, the immune-modulating properties of this MPLA : Ova fusion protein were investigated using mouse bone marrow-derived $\mathrm{mDC}$ and a coculture system of $\mathrm{mDC}$ and allergen-specific DO11.10 CD4 ${ }^{+} \mathrm{T}$ cells ( $\mathrm{mDC}$ : DO11.10 CD4 ${ }^{+} \mathrm{TC}$ ) ex vivo to directly compare what effect fusion of MPLA to Ova would have on the initiated immune response.

\section{Methods}

2.1. Coupling of MPLA and Ova. To activate MPLA (InvivoGen, Toulouse, France), it was dissolved in dried dioxane (Sigma) at a concentration of $50 \mathrm{mM}$ and subsequently incubated at $37^{\circ} \mathrm{C}$. 1,1 $1^{\prime}$-Carbonyldiimidazole (CDI, Sigma, Steinheim, Germany) was added to a final concentration of $0.5 \mathrm{M}$ and the mixture was incubated for $2 \mathrm{~h}$ at $37^{\circ} \mathrm{C}$ with stirring. Finally, dioxane was removed by the addition of diethyl ether and evaporation overnight. To couple the allergen to CDI-activated MPLA, EndoGrade Ovalbumin
(Hyglos, Bernried, Germany) was dissolved in $10 \mathrm{mM}$ sodium borate ( $\mathrm{pH} 8.5$ ) at $2.5 \mathrm{mg} / \mathrm{mL}$ and was subsequently used to dissolve the CDI-activated MPLA. After incubation for $48 \mathrm{~h}$ at $4^{\circ} \mathrm{C}$ with stirring, unconjugated MPLA was removed by extensive dialysis against $\mathrm{PBS}$ at $4^{\circ} \mathrm{C}$ for two days. The resulting MPLA: Ova fusion protein was characterized by SDS-PAGE and dynamic light scattering analysis.

2.2. SDS-PAGE. Chemically conjugated MPLA:Ova was compared to EndoGrade Ovalbumin (Hyglos) by SDS-PAGE according to the method described by Laemmli (cross linker $\mathrm{C}=5 \%$, total bis/acrylamide 15\%) [17] under reducing conditions.

2.3. Dynamic Light Scattering Analysis. Dynamic light scattering analysis was performed using a Zetasizer Nano ZS (Malvern, Herrenberg, Germany). For light scattering analysis, $70 \mu \mathrm{L}$ of MPLA ( $1 \mathrm{mg} / \mathrm{mL})$, MPLA : Ova $(0.6 \mathrm{mg} / \mathrm{mL})$, LPS $(1 \mathrm{mg} / \mathrm{mL})$, or Ova $(1 \mathrm{mg} / \mathrm{mL})$ in PBS was analyzed at room temperature. Three individual measurements per sample were performed and the mean frequencies (calculated as relative $\%$ in class) of hydrodynamic radii $\left(r_{\mathrm{H}}\right)$ in $\mathrm{nm}$ were plotted.

2.4. In Vitro Generation of Mouse Bone Marrow-Derived Dendritic Cells. Mouse myeloid dendritic cells (mDCs) were generated as described previously [18]. Briefly, bone marrow cells (BMCs) were isolated from femur and tibia of BALB/c, mice and differentiated into mDCs using GM-CSF (R\&D Systems, Minneapolis, USA). On day eight, mDCs were harvested for experiments.

2.5. Preparation and Stimulation of $m D C$ and $m D C: D O 11.10$ $C D 4^{+}$T Cell Cocultures. Splenic CD $4^{+} \mathrm{T}$ cells were isolated from Ova-TCR transgenic DO11.10 mice using the CD4 ${ }^{+}$ T Cell Isolation Kit (Miltenyi Biotec, Bergisch Gladbach, Germany). BALB/c mDCs $\left(3.2 \times 10^{5}\right.$ cells $\left./ \mathrm{mL}\right)$ were cultured alone or in combination with DO11.10 CD $4^{+} \mathrm{T}$ cells $(8.0 \times$ $10^{5}$ cells $/ \mathrm{mL},>95 \%$ purity) and stimulated with equimolar amounts of Ova, MPLA, MPLA mixed with Ova (MPLA + Ova), or MPLA : Ova (fusion protein) for $72 \mathrm{~h}$. Subsequently, concentrations of IL-1 $\beta$, IL-2, IL-5, IL-6, IL-9, IL-10, IL-12p70, IL-13, IL-17A, TNF- $\alpha$, and IFN- $\gamma$ in the supernatants were measured by BD OptEIA ELISA (BD Biosciences, Heidelberg, Germany) or Ready-SET-Go! ELISA Sets (eBiosciences, Frankfurt, Germany).

2.6. Statistical Analysis. The hypothesis of a significant higher cytokine secretion among all three concentrations used for stimulation was tested with a two-factorial analysis of variance (ANOVA) with factors stimulus $(0.2,1.0$, and 5.0) and group ("MPLA + OVA" or "MPLA : OVA"). For statistical significant results the following convention was used: ${ }^{*} p$ value $<0.05,{ }^{* *} p$ value $<0.01$, and ${ }^{* * *} p$ value $<0.001$. The statistical analysis was performed with SAS/STAT software, version 9.4, SAS System for Windows. 


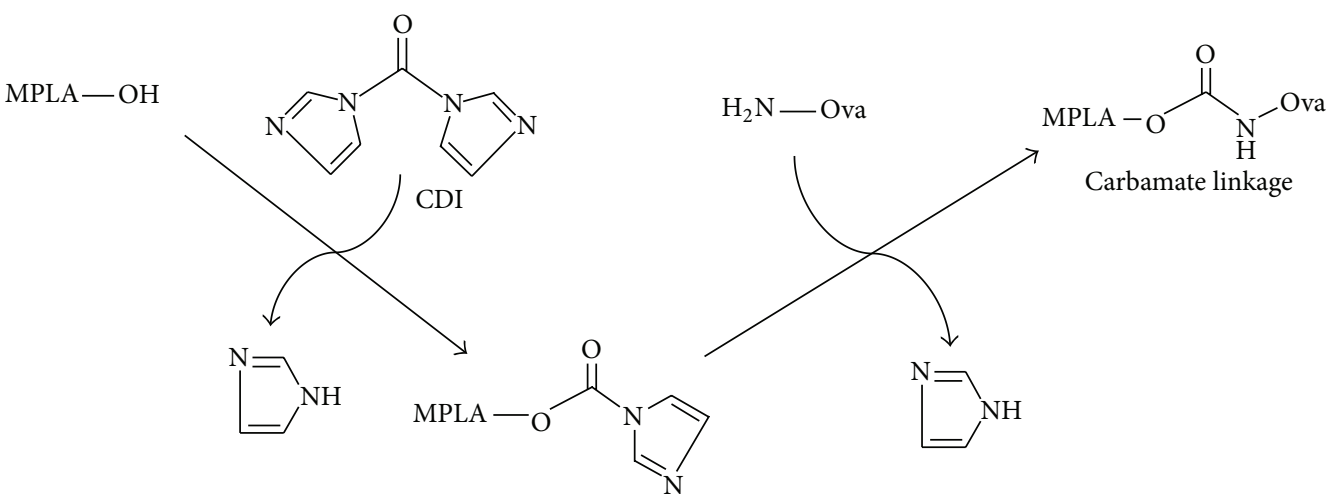

(a)

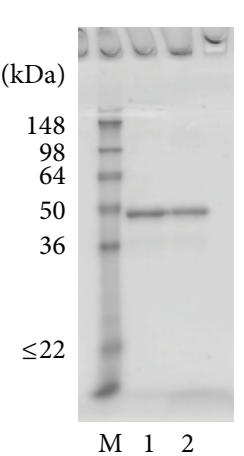

(b)

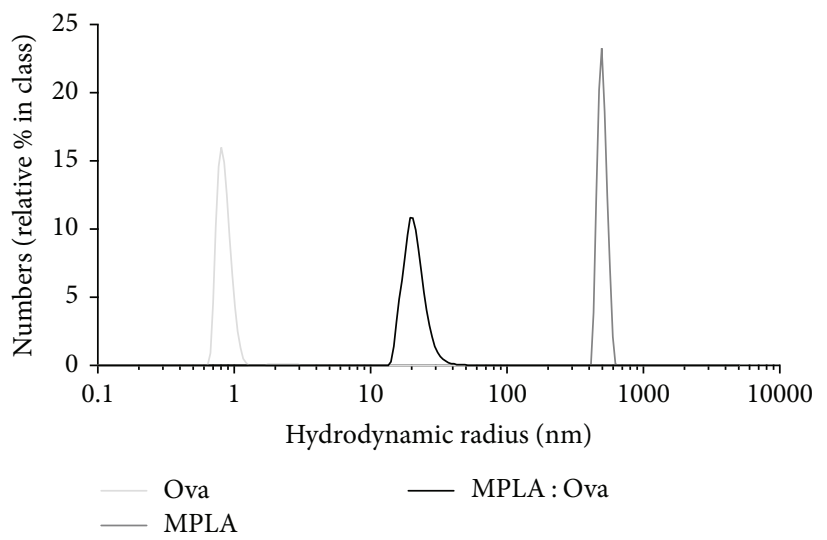

(c)

FIGURE 1: Generation of MPLA : Ova fusion protein. Applied chemical coupling strategy (a). Analysis of MPLA : Ova by reducing SDS-PAGE (b, M: molecular weight marker, 1: EndoGrade Ova, and 2: MPLA : Ova) and dynamic light scattering (c). For light scattering analysis, $70 \mu \mathrm{L}$ of MPLA $(1 \mathrm{mg} / \mathrm{mL})$, MPLA : Ova $(0.6 \mathrm{mg} / \mathrm{mL})$, LPS $(1 \mathrm{mg} / \mathrm{mL})$, or Ova $(1 \mathrm{mg} / \mathrm{mL})$ in PBS was analyzed at room temperature. Three individual measurements per sample were performed and the mean frequencies (calculated as relative $\%$ in class) of hydrodynamic radii $\left(r_{\mathrm{H}}\right)$ in $\mathrm{nm}$ were plotted.

\section{Results}

3.1. A Fusion Protein of MPLA and Ova Shows Noncovalent Aggregation. For the generation of the MPLA : Ova fusion protein, MPLA was conjugated to EndoGrade Ova using a carbonyldiimidazole linker in order to generate a stable carbamate linkage between both molecules (Figure 1(a)). Noncoupled MPLA was removed by extensive dialysis. The resulting MPLA: Ova fusion protein was characterized by SDS-PAGE and displayed a distinct band with an apparent molecular mass of $47 \mathrm{kDa}$ (Figure 1(b)). Compared to Ova (apparent molecular mass of $45 \mathrm{kDa}$ ) this moderate shift of approximately $2 \mathrm{kDa}$ suggests a coupling rate of one molecule of MPLA (molecular mass: $1.7 \mathrm{kDa}$ ) per molecule of Ova (Figure 1(b)).

Dynamic light scattering analysis determined the hydrodynamic radius of MPLA $\left(r_{\mathrm{H}}=496 \mathrm{~nm}\right)$ to be larger than the radius of Ova $\left(r_{\mathrm{H}}=0.9 \mathrm{~nm}\right.$, Figure 1(c)). This finding suggests aggregation in the MPLA preparation which is likely explained by the formation of micelle-like structures by the fatty acid chains of MPLA [19]. Here, the size of aggregates was reduced for the MPLA : Ova fusion protein $\left(r_{\mathrm{H}}=20 \mathrm{~nm}\right)$, likely due to steric hindrance of micelle-formation induced by the fusion of Ova to MPLA (Figure 1(c)). However, compared to Ova alone, the hydrodynamic radius of the MPLA: Ova fusion protein was 22-fold enhanced in size, and no molecules with the hydrodynamic radius of either Ova or MPLA were detected in the MPLA : Ova preparation (Figure 1(c)). Taken together, these findings suggest both a complete coupling of the two molecules at a one-to-one ratio for the fusion protein and a complete removal of noncoupled MPLA by dialysis, resulting in a pure fusion protein preparation.

3.2. MPLA: Ova Boosts mDC-Derived Cytokine Secretion Compared to the Mixture of Both Components. To investigate the potential immune-modulating properties of the fusion protein compared to both components alone or as a mixture we performed stimulation experiments using both myeloid dendritic cells (mDCs) alone (Figure 2(a)) and in coculture experiments with Ova-T cell receptor transgenic DO11.10 $\mathrm{CD}^{+} \mathrm{T}$ cells (Figures 2(b) and 3).

In $\mathrm{mDC}$ cultures, stimulated with the different constructs, application of the MPLA : Ova fusion protein resulted in increased secretion of IL- $1 \beta$, IL-6, IL-10, and TNF- $\alpha$ (Figure 2(a), IL-1 $\beta$ : MPLA : Ova versus MPLA + Ova $p=0.0241$, 

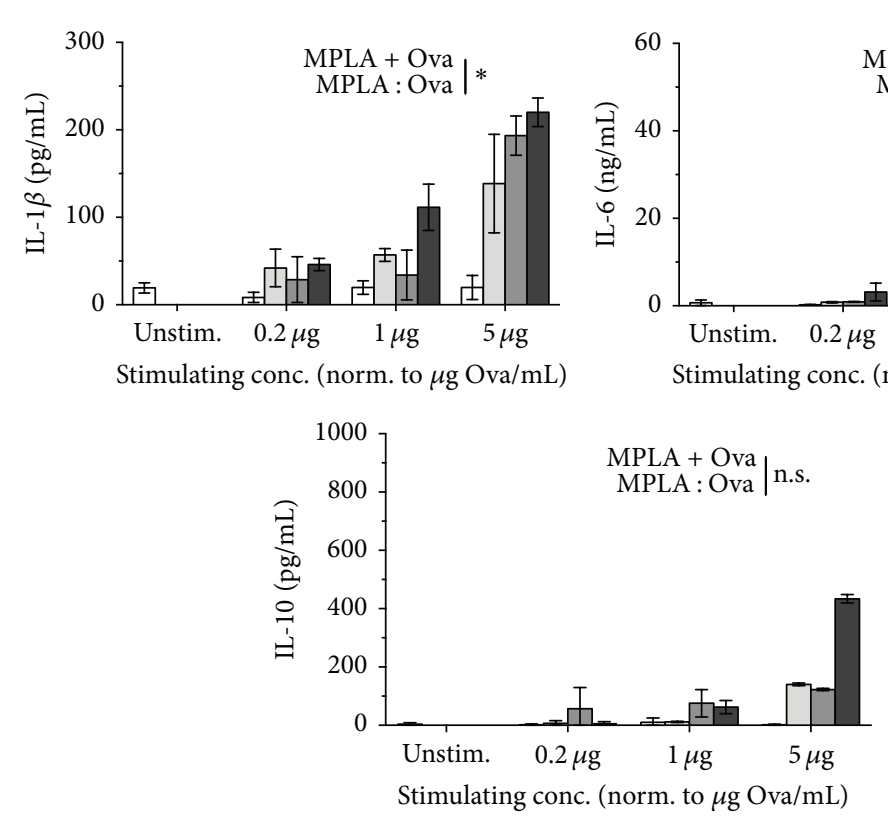
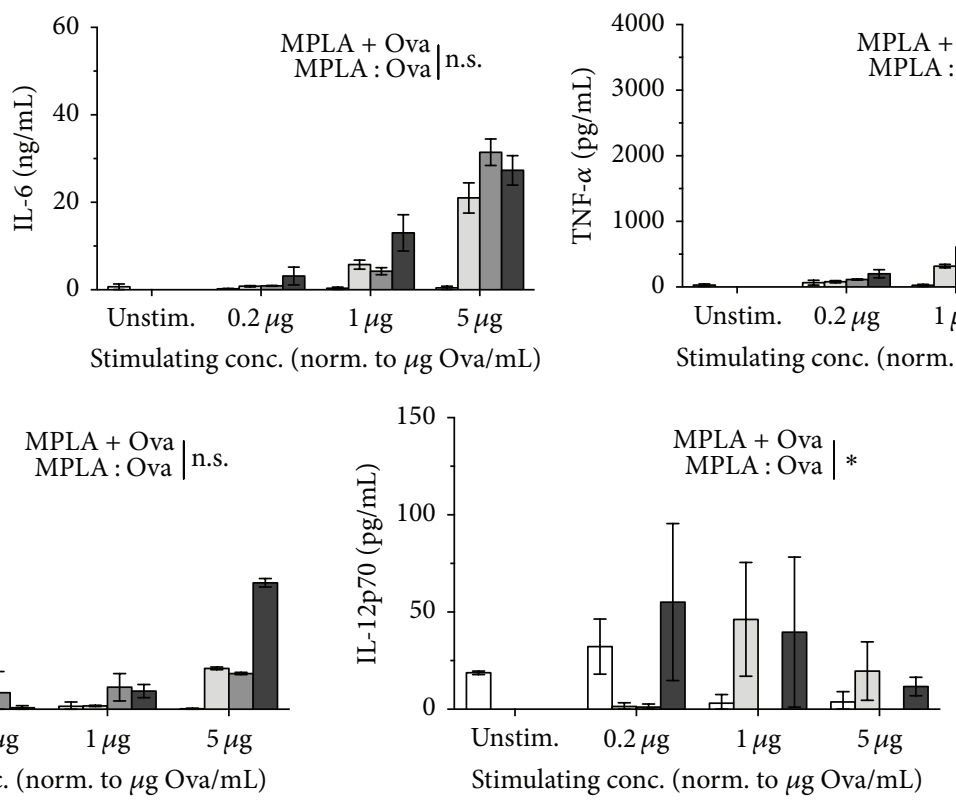
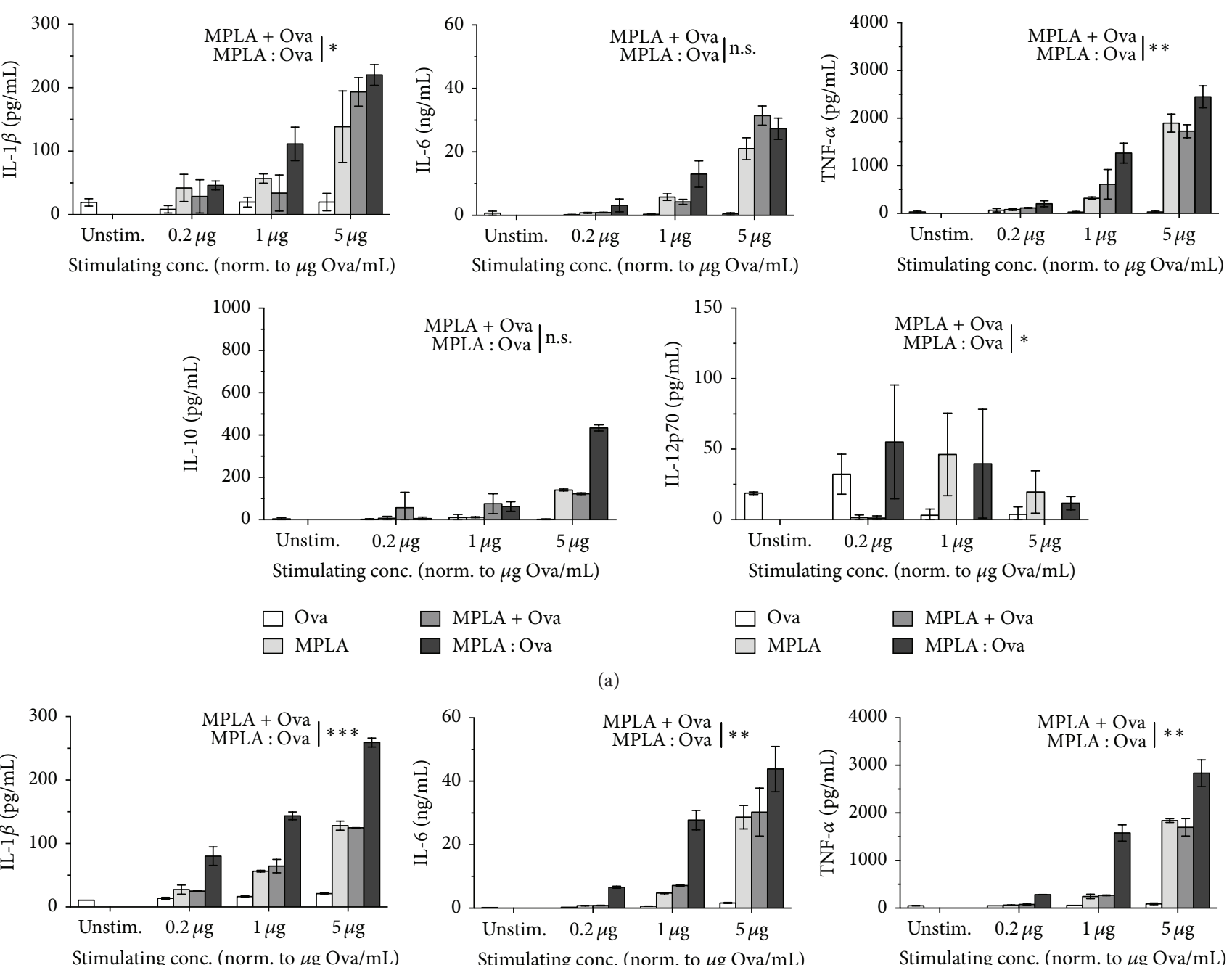

(a)
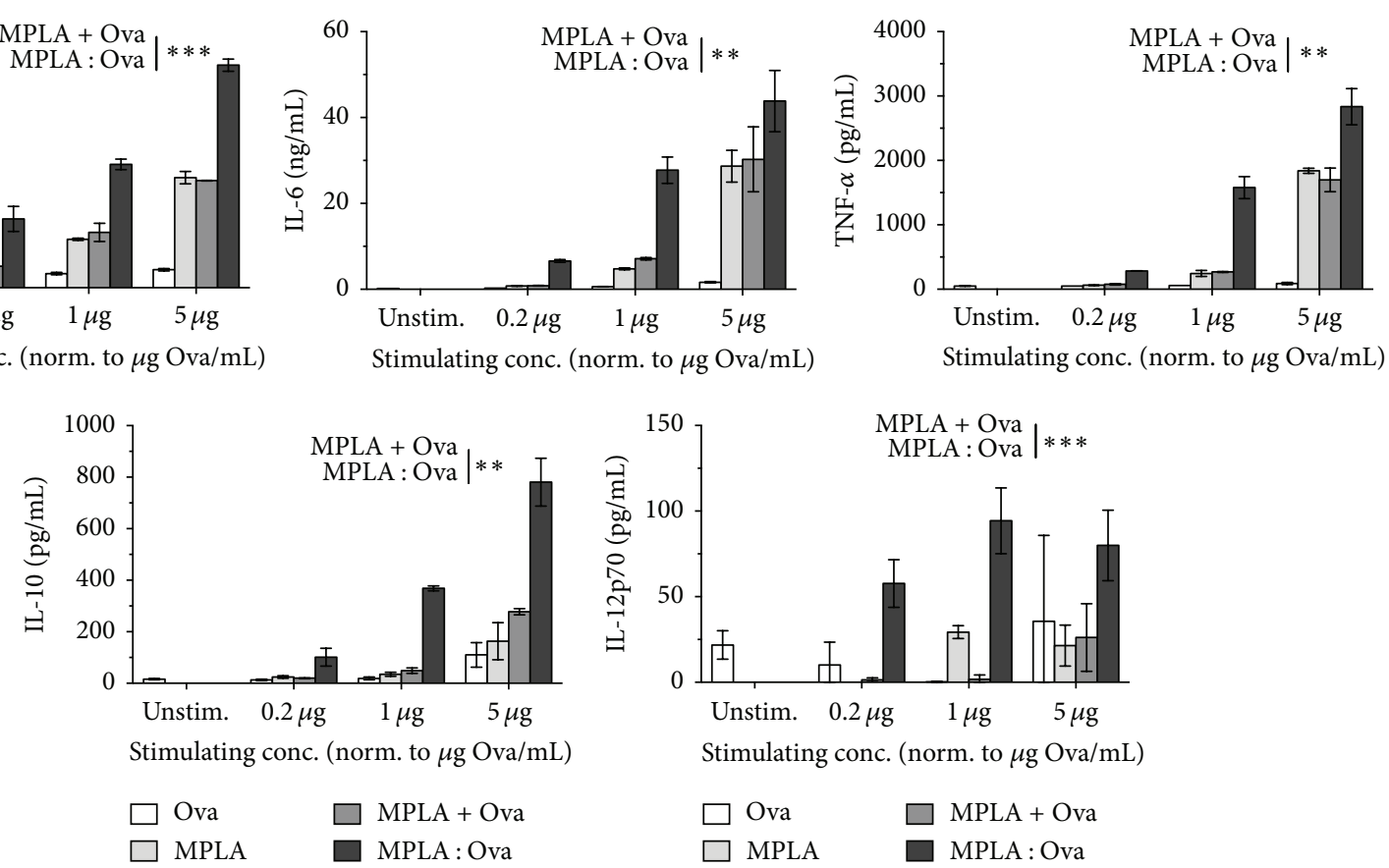

(b)

FIGURE 2: The MPLA : Ova fusion protein boosts mDC-derived cytokine secretion compared to the mixture of both components. Cytokine secretion determined from either BALB/c mDC $\left(3.2 \times 10^{5}\right.$ cells $/ \mathrm{mL}$, a) or BALB/c mDC $\left(3.2 \times 10^{5}\right.$ cells $\left./ \mathrm{mL}\right)$ plus DO11.10 CD4 ${ }^{+} \mathrm{T}$ cell $(8.0 \times$ $10^{5}$ cells $/ \mathrm{mL},>95 \%$ purity) cocultures (b) stimulated with equimolar amounts of Ova (white bars), MPLA (light grey bars), MPLA + Ova (dark grey bars), and the MPLA : Ova fusion protein (black bars) for $72 \mathrm{~h}$ and analyzed by ELISA. ELISAs were performed using either BD OptEIA $^{\mathrm{TM}}$ ELISA (BD Biosciences) or Ready-SET-Go! ELISA Sets (eBiosciences). Data are mean results of two independent experiments \pm $\mathrm{SD}$. The hypothesis of a significant higher cytokine secretion among all three concentrations used for stimulation was tested with a twofactorial analysis of variance (ANOVA) with factors stimulus (0.2, 1.0, and 5.0) and group ("MPLA + OVA" or "MPLA : OVA"). For statistical significant results the following convention was used: ${ }^{*} p$ value $<0.05,{ }^{* *} p$ value $<0.01$, and ${ }^{* * *} p$ value $<0.001$. The statistical analysis was performed with SAS ${ }^{\circledR} /$ STAT software, version 9.4, SAS System for Windows. 

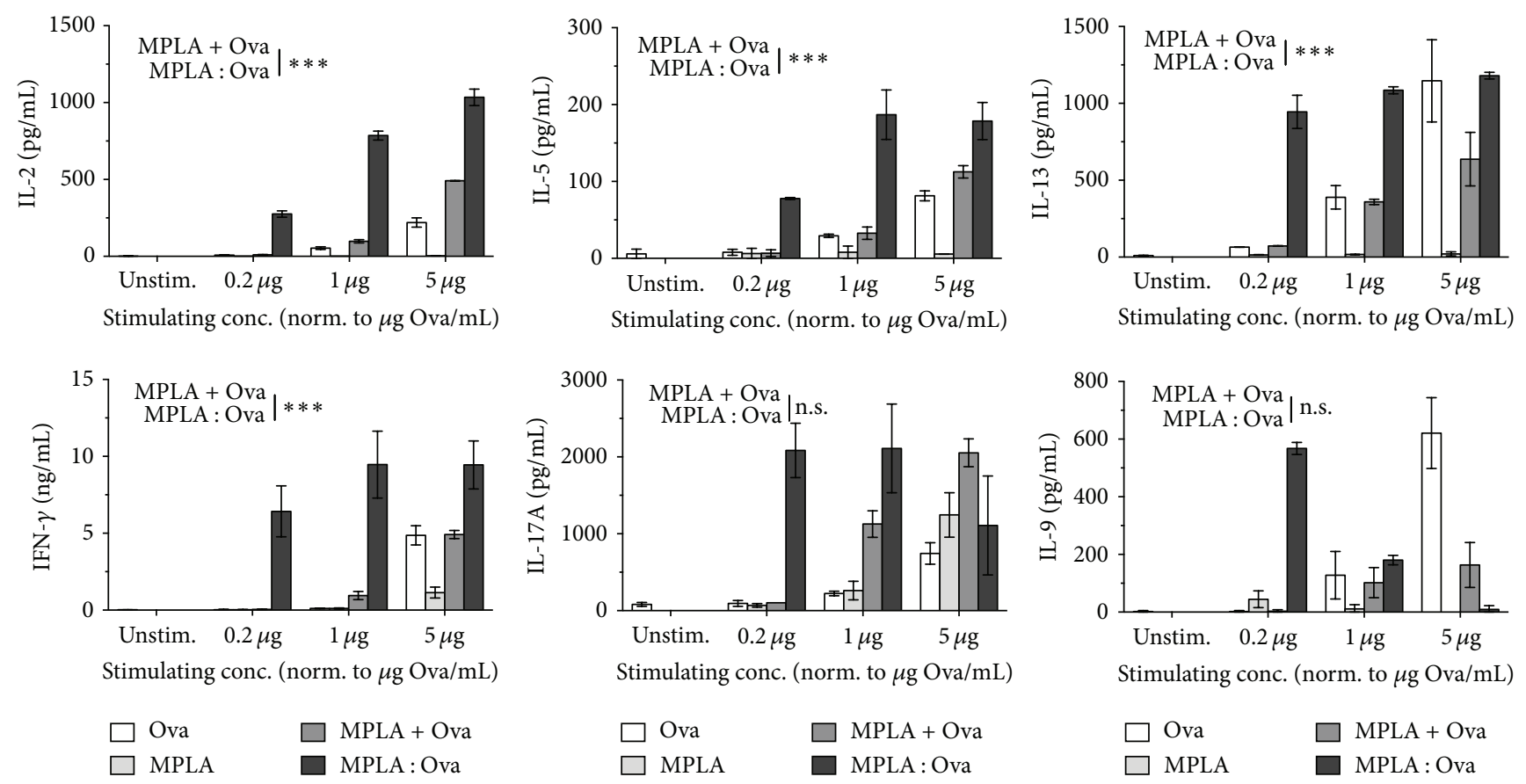

Figure 3: The MPLA : Ova fusion protein nonspecifically boosts Th1, Th2, and Th17 cytokine secretion from Ova-specific T cells. Cytokine secretion from BALB/c mDC $\left(3.2 \times 10^{5}\right.$ cells $\left./ \mathrm{mL}\right)$ and DO11.10 CD4 ${ }^{+} \mathrm{T}$ cell $\left(8.0 \times 10^{5}\right.$ cells $/ \mathrm{mL},>95 \%$ purity $)$ cocultures stimulated with Ova (white bars), MPLA (light grey bars), MPLA + Ova (dark grey bars), or MPLA : Ova (black bars) for either $24 \mathrm{~h}$ (IL-2) or $72 \mathrm{~h}$ (all other cytokines). ELISAs were performed using either BD OptEIA ELISA (BD Biosciences) or Ready-SET-Go! ELISA Sets (eBiosciences). Data are mean results of two independent experiments \pm SD. Statistical analysis was performed according to Figure 2.

IL-6: $p=0.3447$, IL-10: $p=0.2114$, TNF- $\alpha: p=0.0078)$. In contrast, stimulation with MPLA : Ova resulted in a dosedependent decrease of IL-12p70 secretion, which was not observed for either component alone or the mixture of MPLA and Ova (Figure 2(a) IL-12p70: MPLA : Ova versus MPLA + Ova $p=0.0272)$.

Compared to the results obtained when stimulating mDCs alone, the levels of MPLA: Ova-induced cytokine secretion observed upon stimulation of $\mathrm{mDC}: \mathrm{DO} 11.10 \mathrm{CD} 4^{+}$ $\mathrm{T}$ cell cultures were either unchanged (TNF- $\alpha$ and IL$1 \beta$ ) or further increased (IL-6, IL-10, and IL-12p70, Figure 2(a) versus 2(b)). Therefore, when MPLA: Ova was added to $\mathrm{mDC}$ : DO11.10 $\mathrm{CD}^{+}{ }^{+} \mathrm{T}$ cell cultures, the fusion protein induced a significantly higher cytokine secretion than equimolar amounts of either component alone or the mixture of MPLA and Ova (Figure 2(b)). Here, in direct comparison to the mixture of both components, the MPLA : Ova fusion protein significantly boosted both proinflammatory (IL-1 $\beta$ : MPLA : Ova versus MPLA + Ova $p<0.0001$, IL-6: $p=$ 0.0026 , IL-12: $p=0.0001$, TNF- $\alpha: p=0.0015)$ and antiinflammatory (IL-10: $p=0.0016$ ) cytokine secretion.

Moreover, for the concentration corresponding to $1 \mu \mathrm{g}$ Ova per mL, MPLA : Ova induced 4-fold higher IL-1 $\beta$, 4-fold higher IL-6, 6-fold higher TNF- $\alpha$, 8-fold higher IL-10, and 53-fold higher IL-12p70 levels compared to the noncoupled mixture of MPLA + Ova (Figure 2(b)).

3.3. MPLA : Ova Boosts Th1, Th2, and Th17 Cytokine Secretion from Ova-Specific T Cells in a Nonbiased Way. In the next step we investigated the effect of MPLA : Ova-mediated $\mathrm{mDC}$ activation on the differentiation of Ova-specific $\mathrm{CD} 4^{+} \mathrm{T}$ cells (Figure 3). In addition to the significantly increased $\mathrm{mDC}$ derived cytokine secretion (Figure 2), induced by the fusion protein compared to the controls, we observed the same effect for enhanced T cell-derived cytokine secretion in the applied coculture system (Figure 3 ).

In accordance with the results shown in Figure 2, at a stimulating concentration corresponding to $1 \mu \mathrm{g}$ Ova per $\mathrm{mL}$, MPLA : Ova induced an 8-fold higher IL-2, 6-fold higher IL5, 3-fold higher IL-13, 10-fold higher IFN- $\gamma$, 2-fold higher IL-17A, and 2-fold higher IL-9 secretion than the equimolar mixture of both components (Figure 3). Here, neither IL2, IL-5, IL-13, IFN- $\gamma$, and IL-17A nor IL-9 secretion was detectable when $\mathrm{mDCs}$ were stimulated in the absence of Ova-specific $\mathrm{CD}^{+} \mathrm{T}$ cells (data not shown). Remarkably, at low concentrations (equivalent to $0.2 \mu \mathrm{g} / \mathrm{mL}$ Ova) MPLA : Ova induced a 20-fold higher production of IL-17A in comparison to the equimolar mixture of MPLA and Ova, whereas at the highest applied concentration there was no difference between the different stimuli (Figure 3).

Of note, this effect was reversed for MPLA : Ova-induced IL-9 production, where stimulation with increasing amounts of MPLA : Ova resulted in a dose-dependent decrease in IL9 secretion, while Ova alone dose-dependently induced IL-9 secretion (Figure 3, IL-9: MPLA : Ova versus MPLA + Ova $p=0.1691)$.

Finally, the MPLA : Ova fusion protein boosted Th1 (IFN$\gamma$ : MPLA : Ova versus MPLA + Ova $p<0.0001$ and IL-2: $p<$ 0.0001 ), Th2 (IL-5: $p=0.0004$ and IL-13: $p<0.0001$ ), and Th17 cytokine secretion (IL-17A, $p=0.1920$ Figure 3 ) from 
allergen-specific $\mathrm{T}$ cells without skewing the overall immune response in any particular direction.

\section{Discussion}

Herein we describe the generation and immunological characterization of a novel vaccine candidate consisting of the adjuvant MPLA and the model allergen Ovalbumin.

Adjuvant:allergen conjugates have several advantages over simple nonconjugated mixtures of both components: (1) they target the conjugate to the respective immune cells by binding to specific immune receptors (in this case TLR4 which may mediate both proinflammatory signaling and uptake). Upon binding to the target cell they (2) deliver the conjugated allergen to the immune cell in the context of the adjuvant-mediated immune cell activation which may influence allergen uptake, processing, and presentation [20]. Moreover, (3) adjuvant and allergen are simultaneously delivered to the same cell in a fixed molecular ratio, thereby preventing potentially detrimental bystander activation.

For this purpose, MPLA and Ovalbumin were coupled chemically via a stable carbamate linkage and the resulting fusion protein was characterized by SDS-PAGE and light scattering analysis. In SDS-PAGE, MPLA : Ova displayed a slight shift in molecular weight from approximately $45 \mathrm{kDa}$ observed for Ova to approximately $47 \mathrm{kDa}$ observed for MPLA: Ova. This moderate shift of approximately $2 \mathrm{kDa}$ indicates a coupling rate of one molecule of MPLA (molecular mass: $1.7 \mathrm{kDa}$ ) per molecule of Ova.

Successful coupling of both molecules was further confirmed by light scattering analysis. With this assay we were able to demonstrate that the resulting MPLA: Ova fusion protein showed a single peak with a hydrodynamic radius of approximately $20 \mathrm{~nm}$, which represents a 22 -fold increase in size compared to nonconjugated Ova. Additionally, no molecules with the hydrodynamic radius of MPLA or Ova were detected within the MPLA: Ova preparation, demonstrating a complete removal of noncoupled MPLA by dialysis after coupling.

Previous studies investigating the effects of adjuvant:allergen fusion proteins, including TLR5-, TLR7-, and TLR9-ligands, on the modulation of allergen-specific immune responses demonstrated the potential for such conjugate vaccines to improve allergy treatment [13-16]. Studies by Kastenmüller et al. [15] and Filí et al. [16] describe allergen fusion proteins with TLR7- and TLR7/8-ligands as adjuvants.

Kastenmüller and colleagues reported a conjugate vaccine of a TLR7/8-ligand and Ova and showed this conjugate to elicit potent Th1-biased $\mathrm{CD}^{+}{ }^{+}$and $\mathrm{CD} 8^{+} \mathrm{T}$ cell responses by activation and recruitment of dendritic cells to draining lymph nodes and the subsequent induction of type I interferon production [15]. In line with these results, Filí and coauthors described that the mite allergen nDer $\mathrm{p} 2$ conjugated to a TLR7-ligand (4-(6-amino-9benzyl-8-hydroxy-9H-purin-2-ylsulfanyl-)-butyric acid succinimidyl ester) stimulated IL-12 and IFN- $\gamma$ production from monocytes and plasmacytoid DC and reduced allergic symptoms, while inducing allergen-specific $\operatorname{IgG}_{2 \mathrm{~A}}$ antibodies in mice [16]. In this context, no induction of autoantibodies or Th17 cells was observed [16].

Moreover, Tighe and colleagues described the conjugation of a 22-mer CpG-motif, acting as a TLR9-ligand, to the major short ragweed allergen Amb a 1 [14]. In accordance with the results from the other adjuvant:allergen fusion proteins, this conjugate was shown to both induce Th1-biased immune responses in both naive and sensitized mice and suppress IgE-induction after allergen-challenge [14].

In our own preliminary work we could show that prophylactic and therapeutic vaccination with a recombinant conjugate of the TLR5 agonist flagellin A (FlaA) from Listeria monocytogenes and Ova ( $\mathrm{rFlaA}: \mathrm{Ova})$ was able to diminish Th2 responses in a mouse model of Ova-induced intestinal allergy [13]. Cocultures of mouse bone marrow-derived mDCs and $\mathrm{CD}^{+}$DO11.10 T cells demonstrated an IL-10dependent reduction of Th2 and Th1 cytokine production upon stimulation with rFlaA: Ova but not with rOva and FlaA provided as a mixture [21].

When stimulating mDC with MPLA:Ova and the respective controls we observed an increased secretion of both proinflammatory (IL-1 $\beta$, IL- 6 , and TNF- $\alpha$ ) and antiinflammatory (IL-10) cytokines. Here, in direct comparison of $\mathrm{mDC}$ stimulations with $\mathrm{mDC}: \mathrm{CD} 4^{+} \mathrm{TC}$ coculture stimulations, overall levels of MPLA : Ova-induced cytokines were further increased in cocultures compared to the respective stimulation of $\mathrm{mDCs}$ alone. These results suggest that the $\mathrm{mDC}$ : TC interaction in the cocultures either further increased $\mathrm{mDC}$-derived secretion (possibly by licensing effects of $\mathrm{CD}^{+} \mathrm{T}$ cells via mechanisms such as CD40CD40L interaction) or induced additional production of the respective cytokines from Ova-specific $\mathrm{T}$ cells.

Unexpectedly in $\mathrm{mDC}$ : DO11.10 $\mathrm{CD}^{+} \mathrm{T}$ cell cocultures, when chemically fusing the TLR4 agonist MPLA to Ova, we observed a boost of Th 2 cytokine (IL-5 and IL-13) production in such $\mathrm{mDC}$ : DO11.10 $\mathrm{CD}^{+} \mathrm{T}$ cell cocultures compared to equimolar amounts of MPLA + Ova. In addition, we observed an upregulation of both Th1 and Th17 cytokines IFN- $\gamma$ and IL-17A as well as $\mathrm{mDC}$-derived proinflammatory (IL-1 $\beta$, IL-6, and TNF- $\alpha$ ) and anti-inflammatory (IL-10) cytokines. Such strong APC activation and TC-derived cytokine boosts without distinct bias towards a defined $\mathrm{T}$ cell subtype (e.g., Thl-cells) are likely detrimental and can have significant consequences for both vaccine development and safety.

In contrast to this pattern, we observed a dose-dependent decrease of IL-9 secretion upon stimulation with the MPLA : Ova fusion protein while Ova alone or the mixture of MPLA + Ova dose-dependently induced IL-9 secretion. IL-9 stimulates cell growth and prevents apoptosis [21]; therefore, we believe that the observed reduction of IL-9 secretion upon stimulation with higher doses of MPLA : Ova represents a countermeasure to limit excessive cell activation and its potentially detrimental effects by this fusion protein. In line with our results the available literature describes the suppression of TC-derived IL-9 secretion by differentially activated DC: Rampal and colleagues reported that retinoic acid-monocyte-derived dendritic cells in the presence of TGF- $\beta 1$ and IL- 4 inhibited IL- 9 and induced IFN$\gamma$ expression [22]. Concordantly, IFN- $\gamma$ secretion was shown 
to inhibit Th9-differentiation [23]. Taking into account these results, the dose-dependent decrease of IL-9 secretion upon stimulation with the MPLA: Ova fusion protein may also be explained by the strong induction of other cytokines such as IFN- $\gamma$ in higher stimulation concentrations. However, further investigations of this phenomenon and physiological relevance of MPLA : Ova-induced IL-9 secretion will need to be addressed in further in vivo studies.

In contrast to the strongly Th1-promoting TLR7 or TLR9 ligands, LPS was described to induce both Th1 and Th2 responses depending on either the applied dose [24] or the genetic background of the used organism [25]. The influence of genetic background on the capacity of LPS to induce either Th1 or Th2 responses was, for example, investigated by Soudi and colleagues [25]. They found that (in line with the well-described tendency of $\mathrm{C} 57 \mathrm{BL} / 6$ and $\mathrm{BALB} / \mathrm{c}$ to induce Th1 and Th2 responses, resp.) macrophages isolated from thioglycolate stimulated C57BL/6 mice produced more IL-17, IL-10, and IFN- $\gamma$, while BALB/c macrophages produced more TGF- $\beta 1$ and IL- 4 when stimulated with LPS [25].

In our own previous work, when directly comparing LPS and MPLA for their capacity to skew Ova-induced T helper cell differentiation in BALB/c mDC:DO11.10 $\mathrm{CD}^{+}{ }^{+}$TC coculture experiments we have demonstrated that MPLA was able to boost Ova-induced Th2-cytokine (IL-4, IL-5, and IL13) secretion [12]. Interestingly, this effect was not observed upon coapplication of LPS and Ova [12]. Here, further studies are necessary to more clearly define the adjuvant capacity of MPLA in comparison to its parent molecule LPS. Also, while MPLA was shown to induce an immune deviation in favor of Th1 responses in grass pollen allergic donors [26] the differences in MPLAs adjuvant capacity in men versus mice are not yet fully clear and need further investigation. In line with this, the question whether the results obtained for the MPLA : Ova fusion protein in this study can be transferred to human DC: TC cocultures needs to be addressed in further studies.

In summary, we successfully generated a novel fusion protein consisting of the vaccine adjuvant MPLA and the model allergen Ova by chemical linkage. The generated fusion protein displayed a suggested coupling ratio of one molecule MPLA per molecule of Ova and was shown to aggregate, possibly mediated by the formation of micellelike structures by the fatty chains of MPLA. Immunologically we observed that, compared to both components alone or as a mixture, the fusion protein boosted both mDCcytokines as well as TC-derived Th1, Th2, and Th17 cytokine secretion without skewing the induced TC-differentiation in any particular direction.

Although the generated MPLA : Ova fusion protein may not be a suitable vaccine candidate for allergy treatment, due to the nonbiased boost of allergen-specific Th1, Th2, and Th17 responses, these findings open many new avenues for future research in the field of adjuvant biology, allergy, and immunology. Here, MPLA : antigen fusion proteins might hold potential for the treatment of other diseases which require a strong stimulation of the hosts immune system (e.g., cancer).

\section{Conclusions}

(i) A fusion protein of the TLR4-ligand MPLA and Ovalbumin (MPLA : Ova) was generated in a highly pure form with a coupling ratio of one molecule MPLA per molecule of Ova and without contaminations by either noncoupled MPLA or Ova.

(ii) Immunologically, in $\mathrm{mDC}$ : DO11.10 $\mathrm{CD} 4^{+} \mathrm{TC}$ cocultures MPLA: Ova induced both stronger innate $(\mathrm{mDC})$ and adaptive (Ova-specific TC) immune responses compared to the mixture of both components, boosting Th1, Th2, and Th17 TC-derived cytokine secretion.

\section{Disclosure}

Adam Flaczy current address is School of Medicine and Medical Science, University College Dublin, Belfield, Dublin, Ireland.

\section{Competing Interests}

In the past 3 years Stefan Vieths has received speakers honoraria by the Swiss Society of Allergy and Clinical Immunology, the German Society of Dermatology, and by the "Ärzteverband Deutscher Allergologen," which may be perceived as potential conflict of interests. All other authors have no conflict of interests to declare.

\section{Acknowledgments}

The authors would like to thank Maren Krause, Paul-EhrlichInstitut, for breeding of DO11.10 mice. This work was funded in part by the German Research Foundation (SCHE637/3).

\section{References}

[1] J. L. Justicia, V. Cardona, P. Guardia et al., "Validation of the first treatment-specific questionnaire for the assessment of patient satisfaction with allergen-specific immunotherapy in allergic patients: the ESPIA questionnaire," Journal of Allergy and Clinical Immunology, vol. 131, no. 6, pp. 1539-1546.e2, 2013.

[2] R. Valenta, "The future of antigen-specific immunotherapy of allergy," Nature Reviews Immunology, vol. 2, pp. 446-453, 2002.

[3] D. Toussi and P. Massari, "Immune adjuvant effect of molecularly-defined toll-like receptor ligands," Vaccines, vol. 2, no. 2, pp. 323-353, 2014.

[4] J. T. Ulrich and K. R. Myers, "Monophosphoryl lipid A as an adjuvant. Past experiences and new directions," Pharmaceutical Biotechnology, vol. 6, pp. 495-524, 1995.

[5] M. Kundi, "New hepatitis B vaccine formulated with an improved adjuvant system," Expert Review of Vaccines, vol. 6, no. 2, pp. 133-140, 2007.

[6] S. T. Agnandji, B. Lell, S. S. Soulanoudjingar et al., "First results of phase 3 trial of RTS,S/AS01 malaria vaccine in African children," The New England Journal of Medicine, vol. 365, pp. 1863-1875, 2011. 
[7] B. Romanowski, P. C. de Borba, P. S. Naud et al., "Sustained efficacy and immunogenicity of the human papillomavirus (HPV)16/18 AS04-adjuvanted vaccine: analysis of a randomised placebo-controlled trial up to 6.4 years," The Lancet, vol. 374, no. 9706, pp. 1975-1985, 2009.

[8] P. Patel and A. M. F. Salapatek, "Pollinex ${ }^{\circledR}$ quattro: a novel and well-tolerated, ultra short-course allergy vaccine," Expert Review of Vaccines, vol. 5, no. 5, pp. 617-629, 2006.

[9] C. R. Casella and T. C. Mitchell, "Putting endotoxin to work for us: monophosphoryl lipid a as a safe and effective vaccine adjuvant," Cellular and Molecular Life Sciences, vol. 65, no. 20, pp. 3231-3240, 2008.

[10] N. Mothes, M. Heinzkill, K. J. Drachenberg et al., "Allergenspecific immunotherapy with a monophosphoryl lipid Aadjuvanted vaccine: Reduced seasonally boosted immunoglobulin E production and inhibition of basophil histamine release by therapy-induced blocking antibodies," Clinical and Experimental Allergy, vol. 33, no. 9, pp. 1198-1208, 2003.

[11] P. Baldrick, D. Richardson, G. Elliott, and A. W. Wheeler, "Safety evaluation of monophosphoryl lipid A (MPL): an immunostimulatory adjuvant," Regulatory Toxicology and Pharmacology, vol. 35, no. 3, pp. 398-413, 2002.

[12] S. Schülke, A. Flaczyk, L. Vogel et al., "MPLA shows attenuated pro-inflammatory properties and diminished capacity to activate mast cells in comparison with LPS," Allergy: European Journal of Allergy and Clinical Immunology, vol. 70, no. 10, pp. 1259-1268, 2015.

[13] S. Schülke, M. Burggraf, Z. Waibler et al., "A fusion protein of flagellin and ovalbumin suppresses the $\mathrm{TH} 2$ response and prevents murine intestinal allergy," Journal of Allergy and Clinical Immunology, vol. 128, no. 6, pp. 1340.e12-1348.e12, 2011.

[14] H. Tighe, K. Takabayashi, D. Schwartz et al., "Conjugation of immunostimulatory DNA to the short ragweed allergen Amb a 1 enhances its immunogenicity and reduces its allergenicity," Journal of Allergy and Clinical Immunology, vol. 106, no. 1 I, pp. 124-134, 2000.

[15] K. Kastenmüller, U. Wille-Reece, R. W. B. Lindsay et al., "Protective $\mathrm{T}$ cell immunity in mice following protein-TLR7/8 agonistconjugate immunization requires aggregation, type I IFN, and multiple DC subsets," The Journal of Clinical Investigation, vol. 121, no. 5, pp. 1782-1796, 2011.

[16] L. Filí, A. Vultaggio, E. Cardilicchia et al., "A novel allergenadjuvant conjugate suitable for specific immunotherapy of respiratory allergy," Journal of Allergy and Clinical Immunology, vol. 132, no. 1, pp. 84-92, 2013.

[17] U. K. Laemmli, "Cleavage of structural proteins during the assembly of the head of bacteriophage T4," Nature, vol. 227, no. 5259, pp. 680-685, 1970.

[18] S. Schülke, Z. Waibler, M.-S. Mende et al., "Fusion protein of TLR5-ligand and allergen potentiates activation and IL-10 secretion in murine myeloid DC," Molecular Immunology, vol. 48, no. 1-3, pp. 341-350, 2010.

[19] P. N. Domadia, A. Bhunia, A. Ramamoorthy, and S. Bhattacharjya, "Structure, interactions, and antibacterial activities of MSI594 derived mutant peptide MSI-594F5A in lipopolysaccharide micelles: role of the helical hairpin conformation in outermembrane permeabilization," Journal of the American Chemical Society, vol. 132, no. 51, pp. 18417-18428, 2010.

[20] S. Khan, M. S. Bijker, J. J. Weterings et al., "Distinct uptake mechanisms but similar intracellular processing of two different toll-like receptor ligand-peptide conjugates in dendritic cells,"
The Journal of Biological Chemistry, vol. 282, no. 29, pp. 2114521159, 2007.

[21] L. Knoops and J.-C. Renauld, "IL-9 and its receptor: from signal transduction to tumorigenesis," Growth Factors, vol. 22, no. 4, pp. 207-215, 2004.

[22] R. Rampal, A. Awasthi, and V. Ahuja, "Retinoic acid-primed human dendritic cells inhibit Th9 cells and induce Th1/Th17 cell differentiation," Journal of Leukocyte Biology, 2016.

[23] G. Murugaiyan, V. Beynon, A. P. Da Cunha, N. Joller, and H. L. Weiner, "IFN- $\gamma$ limits Th9-mediated autoimmune inflammation through dendritic cell modulation of IL-27," The Journal of Immunology, vol. 189, no. 11, pp. 5277-5283, 2012.

[24] J. D. de Boer, J. J. T. H. Roelofs, A. F. de Vos et al., "Lipopolysaccharide inhibits Th2 lung inflammation induced by house dust mite allergens in mice," American Journal of Respiratory Cell and Molecular Biology, vol. 48, no. 3, pp. 382-389, 2013.

[25] S. Soudi, A. Zavaran-Hosseini, Z. M. Hassan, M. Soleimani, F. J. Adegani, and S. M. Hashemi, "Comparative study of the effect of LPS on the function of BALB/c and C57BL/ 6 peritoneal macrophages," Cell Journal, vol. 15, no. 1, pp. 45-54, 2013.

[26] F. Puggioni, S. R. Durham, and J. N. Francis, "Monophosphoryl lipid $\mathrm{A}\left(\mathrm{MPL}^{\circledR}\right)^{*}$ promotes allergen-induced immune deviation in favour of Th1 responses," Allergy: European Journal of Allergy and Clinical Immunology, vol. 60, no. 5, pp. 678-684, 2005. 


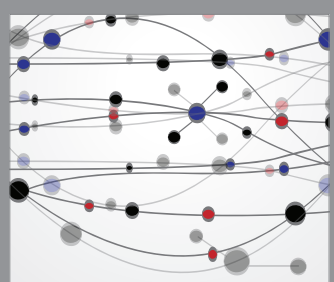

The Scientific World Journal
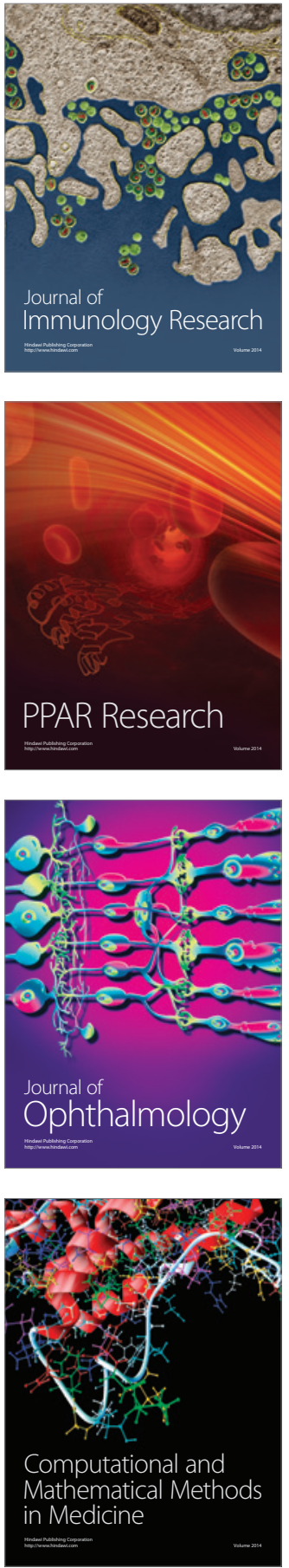

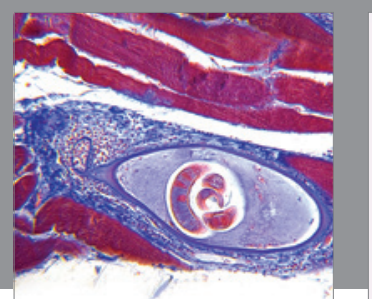

Gastroenterology Research and Practice

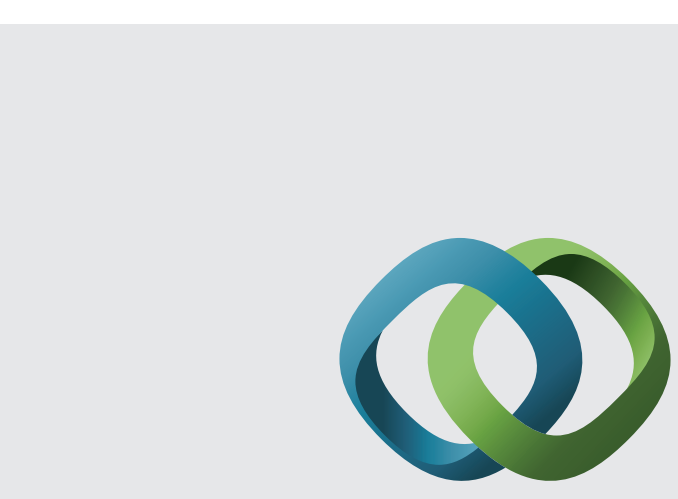

\section{Hindawi}

Submit your manuscripts at

http://www.hindawi.com
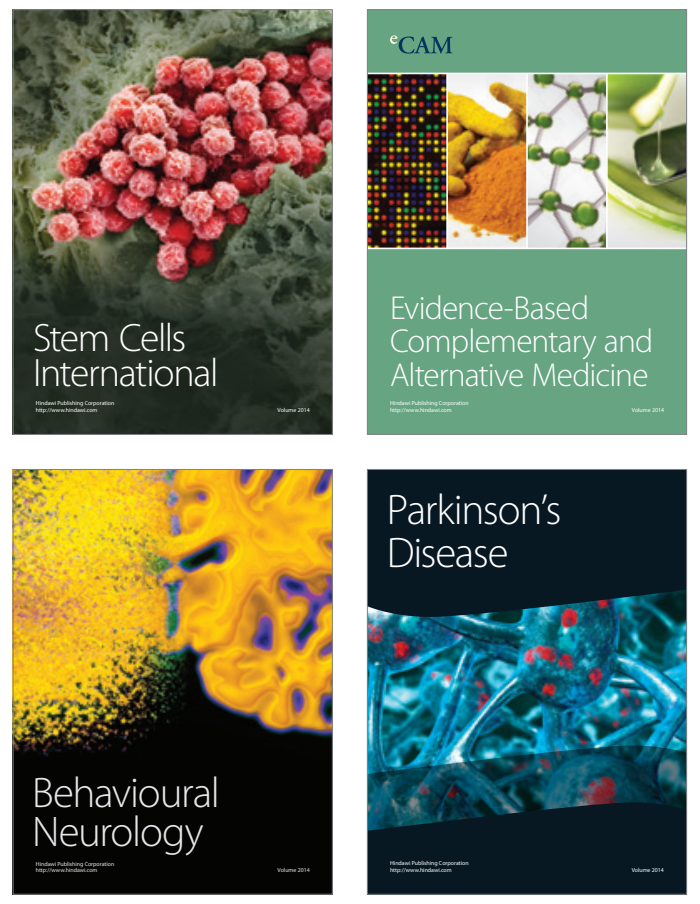
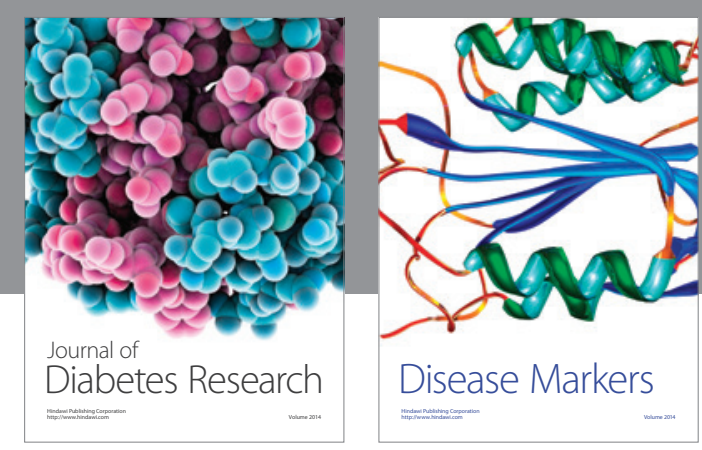

Disease Markers
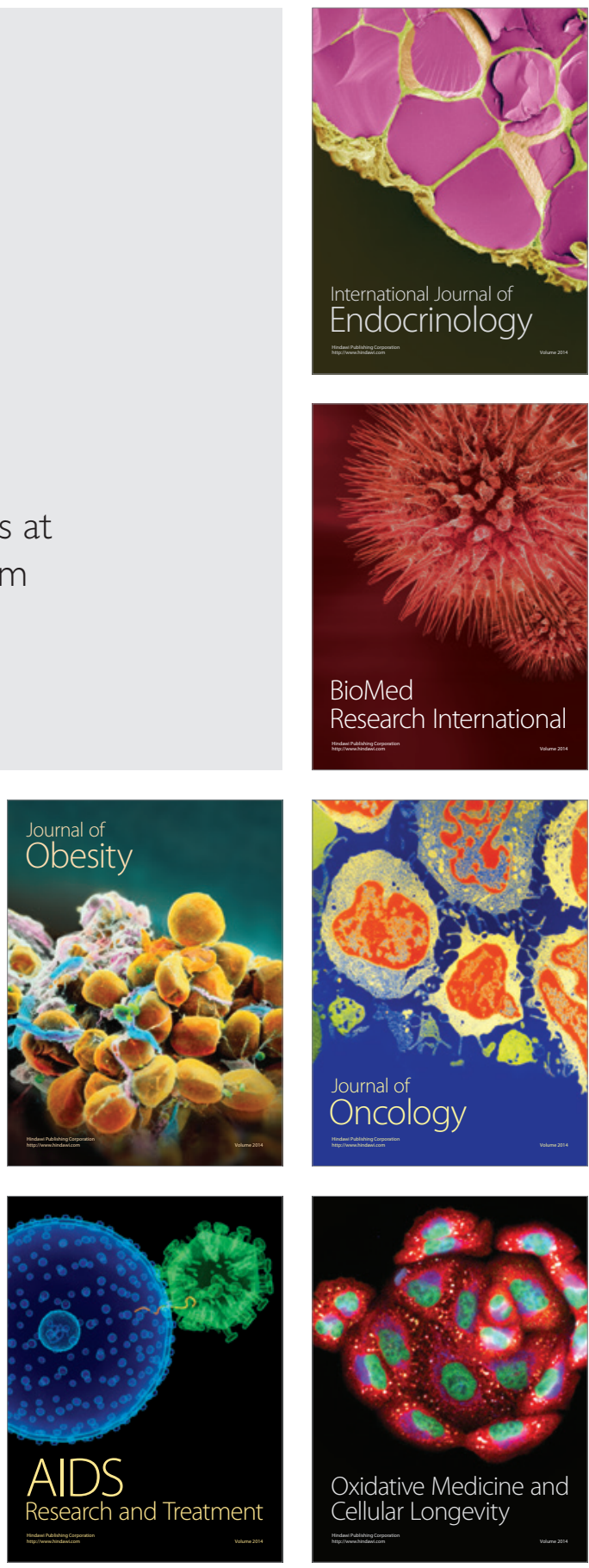